\title{
Leishmaniasis cutánea simuladora de pericondritis
}

\section{Cutaneous leishmania mimicking perichondritis}

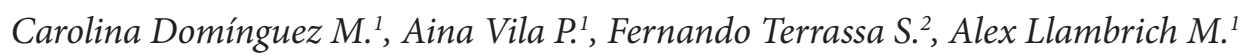

\begin{abstract}
Resumen
La leishmaniasis cutánea presenta una clínica muy característica de pápula eritemato-amarillenta con costra central, en regiones expuestas, que los profesionales de la salud podemos identificar y tratar sin necesidad de biopsia. En ocasiones, se presenta con una clínica atípica que puede conducir a un error diagnóstico, con su perpetuación y malestar en el paciente. Presentamos el caso de una mujer de 53 con una placa eritemato-exudativa por toda la extensión del pabellón auricular izquierdo de varios meses de evolución. Había sido tratada desde el inicio con antibioterapia y corticoides, sin conseguir mejoría y con un claro empeoramiento, al haberse extendido por toda la oreja. Tras la realización de la biopsia, se llegó al diagnóstico de leishmaniasis cutánea erisipeloide. Se procedió a realizar tratamiento dirigido con fluconazol y posteriores infiltraciones de antimoniato de meglumina consiguiendo su completa resolución.

Palabras clave: leishmania cutánea atípica, antimoniato de meglumina intralesional, pericondritis.
\end{abstract}

\begin{abstract}
Cutaneous leishmaniasis presents a very characteristic clinic of erythematous-yellowish papule with central crust, in exposed regions, that we health professionals can identify and treat without the need for a biopsy. Sometimes, it presents with an atypical clinic that can lead to a diagnostic error, with its perpetuation and discomfort in the patient. We present the case of a 53-year-old woman with an erythematous-exudative plaque throughout the extension of the left atrial pavilion of several months of evolution. It had been treated from the start with antiobiotherapy and corticosteroids, without achieving improvement and with a clear worsening, as it had spread throughout the ear. After the biopsy was performed, the diagnosis of erysipeloid cutaneous leishmaniasis was reached. Directed treatment with fluconazole and subsequent infiltrations of meglumine antimoniate were made, achieving its complete resolution. Keywords: atypic cutaneous leishmania, intralesional meglumine antimoniate, perichondritis.
\end{abstract}

'Servicio de Dermatología, Hospital Universitario de Son Llàtzer, Palma de Mallorca. Illes Balears, España. ${ }^{2}$ Servicio de Anatomía Patológica, Hospital Universitario de Son Llàtzer, Palma de Mallorca. Illes Balears, España.

Los autores declaran no tener conflictos de interés.

Recibido el 1 de mayo de 2020. Aceptado el 7 de octubre de 2020.

Correspondencia: Carolina Domínguez M. Servicio de Dermatología Hospital Universitario de Son Llàtzer

Calle Sobreposats, $n^{\circ} 6$, bloque C, piso 3D, 07014. Palma de Mallorca. Islas Baleares, España. Email: caroldguezmah@ hotmail.com

\section{Introducción}

La leishmaniasis cutánea (LC) es una parasitosis por protozoos del género Leishmania, que aparece tras la picadura del mosquito portador de la parasitosis. Su clínica habitual se manifiesta como una pápula o nódulo eritemato-amarillento con costra central en la zona de la picadura. Sin embargo, también existen formas de presentación atípicas, que suponen un reto diagnóstico. Reportamos un caso de leishmaniasis atípica con forma erisipeloide a nivel de pabellón auricular, en la isla de Mallorca. El caso fue revisado por el comité de ética para su divulgación.

\section{Caso Clínico}

Mujer de 53 años, natural de Alemania, que vive en Palma de Mallorca desde hace varios años, sin antecedentes patológicos de interés. Consultó por presentar desde hace más de 6 meses, lesión dolorosa en pabellón auricular, sin otra sintomatología acompañante. A la exploración destaca una placa eritemato-exudativa por toda la extensión del pabellón auricular izquierdo, de consistencia blanda por los bordes del hélix e indurada en su zona más central que correspondía al lóbulo y antitrago (Figuras 1A y B).

La paciente negaba traumatismo previo 
o picadura de insecto en dicha localización, tampoco presentaba otra clínica sistémica acompañante. Acudía con diagnóstico de pericondritis auricular, habiendo realizado múltiples tratamientos con antibióticos y corticoides, orales y tópicos, sin mejoría. Había sido visitada por múltiples especialistas (atención primaria y cirugía plástica), sin encontrarse el otorrinolaringólogo entre ellos. Ante el fracaso terapéutico que se prolongó por 6 meses, fue finalmente derivada a dermatología.

A nuestra visita y con la clínica descrita previamente, el diagnóstico diferencial abar-

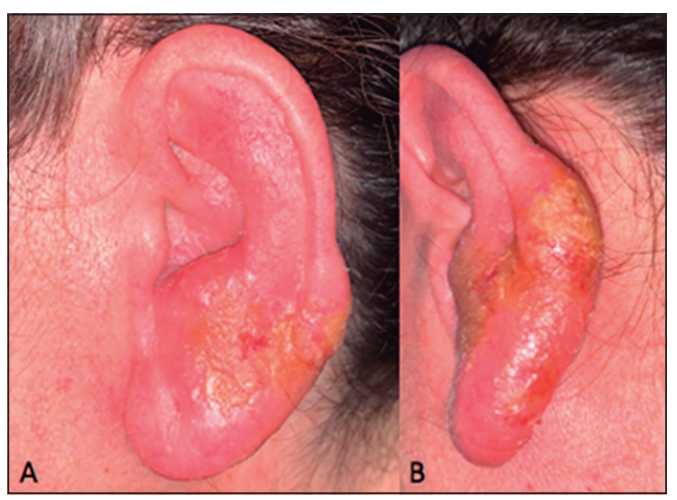

Figura 1. Imagen del pabellón auricular. Se objetiva pabellón auricular izquierdo aumentado de tamaño con respecto a pabellón auricular derecho. Presenta eritema y edema en toda su extensión, exudación amarillenta en región del lóbulo (A) y tercio medio inferior de hélix (B). caba la leishmaniasis cutánea, la sarcoidosis y el linfoma cutáneo. Se procedió a realizar estudio histopatológico que reveló una dermatitis granulomatosa no necrosante con presencia de estructuras puntiformes basófilas intracitoplasmáticas dentro de los histiocitos, que correspondían a amastigotes y que nos aportaban el diagnóstico de leishmania.

Se completó el estudio con análisis sanguíneo, que no evidenció alteraciones ni en el hemograma ni en la bioquímica, y la serología para leishmania y virus de inmunodeficiencia humano fue negativa. Se realizó un electrocardiograma que no evidenció alteraciones del QT, ni arritmias y una ecografía abdominal no patológica. Con estos resultados, el diagnóstico fue de leishmania cutánea de características erisipeloides. Cumpliendo con los criterios de tratamiento sistémico (localización compleja, afectación $>3 \mathrm{~cm}$ ), se le propuso tratamiento con antimoniato de meglumina sistémico, que fue rechazado por la paciente al requerir ingreso. Se inició, por lo tanto, fluconazol 200 $\mathrm{mg}$ /día durante 6 semanas, consiguiendo una gran mejoría y disminución del dolor, pero no su resolución (Figura 2A). Tras la disminución del edema y la extensión de la afectación, se planteó tratamiento intralesional con antimoniato de meglumina. Tras dos infiltraciones, separadas cada una de ellas por dos semanas, se consiguió completa resolución (Figura 2B).

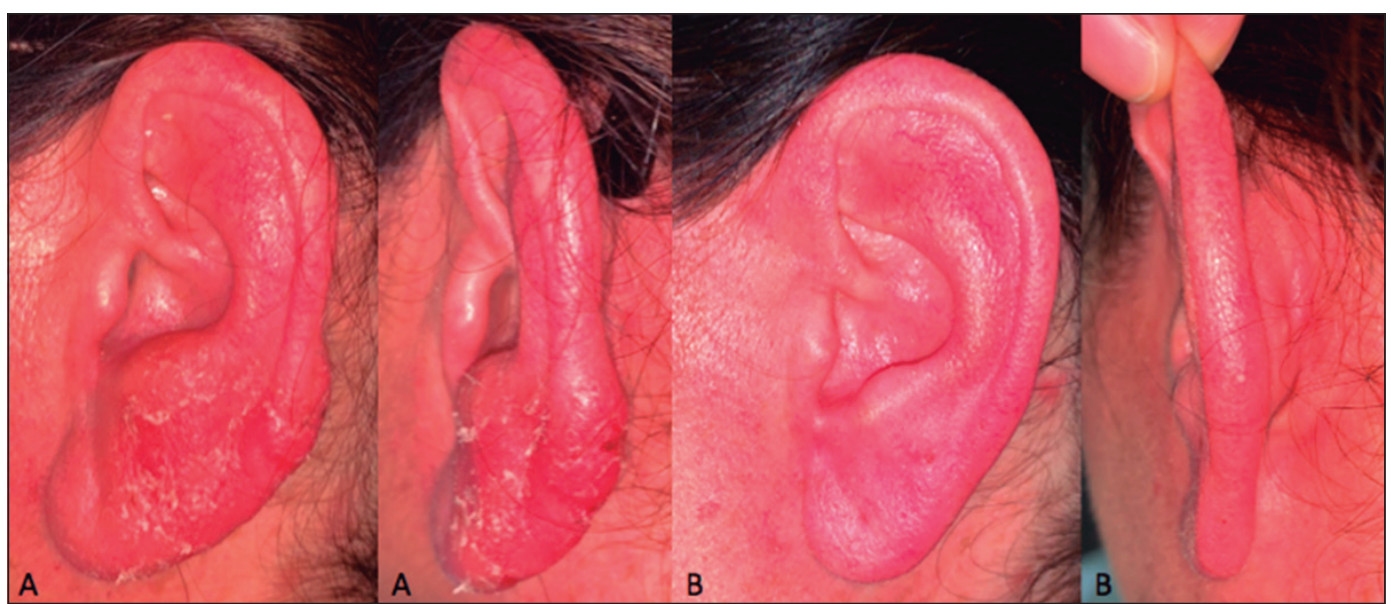

Figura 2. A: Tras 6 semanas de tratamiento con fluconazol, se aprecia mejoría del edema y resolución de la exudación. Persiste el eritema y la induración en lóbulo y parte media del hélix. B: Tras 2 infiltraciones intralesionales de antimoniato de meglumina, presenta resolución completa con recuperación del tamaño previo del pabellón auricular. 


\section{Discusión}

Las leishmaniasis son un grupo de enfermedades producidas por la infección de más de 20 especies de protozoos del género Leishmania. En función de la localización geográfica se distinguen dos grupos: la leishmania del viejo mundo (Mediterráneo, Asia y África) y la leishmania del nuevo mundo (México, América Central y Sudamérica). Actualmente los países sudamericanos más afectadas son Brasil y Colombia, pudiendo traer casos importados a Chile. Su reservorio habitual son los mamíferos domésticos como el perro, el gato, la rata, la liebre y el conejo.

La presentación clínica dependerá de la patogenicidad de la especie involucrada y de la respuesta inmunológica del huésped. Su clínica habitual se manifiesta con una pápula o nódulo eritemato-amarillento con costra central en la zona de la picadura ${ }^{2}$. Sin embargo, también existen formas de presentación atípicas, que suponen un reto diagnóstico. De todas las LC, se ha objetivado que un $5,7 \%$ de los casos van a presentar una forma atípica, a parte de la presentada en el caso clínico, existen muchas otras: lupoide, esporotricoide, paroniquia, palpebral, psoriasiforme, micetoma, chancriforme, cicatricial, zosteriforme, palmo-plantar, verrugas y eccema ${ }^{3}$. Además, dentro de las presentaciones atípicas existe una con afectación preferente del pabellón auricular, característica de los recolectores del caucho, que en un alto porcentaje produce una lesión ulcerada y es denominada "úlcera del chiclero", siendo muy infrecuente en el viejo mundo donde es ocasionada por $L$. mexicana y transmitida por Lutzomyia olmeca, en el nuevo mundo 4 .

La LC erisipeloide, generalmente ocurre en cabeza y tronco superior, siendo su clínica característica una placa eritematoedematosa con leve descamación en superficie sin presencia de ulceración ${ }^{5}$. Se han descrito casos en Pakistán, Turquía, Túnez ${ }^{6}$, Granada y recientemente en Fuenlabrada. Su incidencia está entre los rangos de $0,05 \%$ a $3,2 \%^{7}$, siendo más frecuentes en mujeres de mayor edad y tras ser expuesta la zona a un trauma. Por su localización y su morfología estas lesiones generan un amplio diagnóstico diferencial: celulitis/erisipela, pericondritis, infección fúngica, sarcoidosis, sífilis, eccema, linfoma o metástasis cutánea ${ }^{8}$. Con un diagnóstico diferencial tan amplio, es normal que la paciente pueda ser derivada a distintos especialistas como dermatólogos, otorrinolaringólogos y cirujanos plásticos, como en el caso de nuestra paciente. La sospecha de diagnóstico deberá ser clínica y se deberá confirmar mediante técnicas microbiológicas (cultivo de Leishmania en medios especiales, examen directo, PCR en tejido y/o sangre) y/o histológicas.

Hay múltiples opciones terapéuticas, en función del tamaño y localización se optará por tratamientos tópicos (radiofrecuencia, crioterapia, antimoniato de meglumina intralesional) u orales (fluconazol, metronidazol, antimoniato de meglumina intramuscular $)^{9}$ : si la afectación es múltiple, produce afectación sistémica grave, afecta a regiones con graves secuelas estéticas de la cara o de gran extensión $(>3 \mathrm{~cm})$. El antimoniato de meglumina intramuscular a dosis de $10 \mathrm{mg} / \mathrm{kg} / 12 \mathrm{~h}$ sigue siendo el tratamiento estándar de elección si cumple con los criterios descritos.

\section{Conclusión}

Se presenta el caso clínico de una mujer con una lesión en pabellón auricular de varios meses de evolución, diagnosticada de pericondritis, siendo resistente a tratamientos con antibióticos orales y con empeoramiento progresivo. Tras el examen histológico se confirma LC y se procede a realizar los tratamientos adecuados en cada fase de la patología con su resolución. Por lo que, concluimos que ante pacientes con lesiones sugestivas de pericondritis o erisipela/celulitis que no responden a antibioterapia convencional, hemos de valorar la LC en el diagnóstico diferencial inicial.

\section{Bibliografía}

1. Tarkan Ö, Çetık F, Uzun S. Auricular cutaneous leishmaniasis mimicking neoplastic disease. $J$ Laryngol Otol. 2012;126(8):821-824. doi: 10.1017/ S0022215112001120.

2. Meireles CB, Maia LC, Soares GC, et al. Atypical presentations of cutaneous leishmaniasis: A systematic review. Acta Trop. 2017;172:240-254. doi: 10.1016/j.actatropica.2017.05.022 


\section{CASO CLÍNICO}

3. Novoa Juiz V, Redondo Luciañez R. Mucocutaneous leishmaniasis. Leishmaniasis mucocutánea. Acta Otorrinolaringol Esp. 2016;67(6):355. doi: 10.1016/j. otorri.2015.11.004.

4. Vaira F, Nazzaro G, Pesapane F, Veraldi S. 'Dumbo' ear. Clin Exp Dermatol. 2014;39(5):667-668. doi: 10.1111/ced.12383.

5. Ozdemir M, Cimen K, Mevlito lu I. Post-traumatic erysipeloid cutaneous leishmaniasis. Int J Dermatol. 2007;46(12):1292-1293. doi: 10.1111/j.13654632.2007.03034.x.

6. Mnejja M, Hammami B, Chakroun A, et al. Unusual form of cutaneous leishmaniasis: erysipeloid form. Eur Ann Otorhinolaryngol Head Neck Dis.
2011;128(2):95-97. doi: 10.1016/j.anorl.2010.09.008.

7. Masmoudi A, Ayadi N, Boudaya S, et al.

Polymorphisme clinique de la leishmaniose cutanée du centre et sud tunisien [Clinical polymorphism of cutaneous leishmaniasis in centre and south of Tunisia]. Bull Soc Pathol Exot. 2007;100(1):36-40.

8. Karincaoglu Y, Esrefoglu M, Ozcan H. Atypical clinical form of cutaneous leishmaniasis: erysipeloid form. Int J Dermatol. 2004;43(11):827-829. doi: 10.1111/j.1365-4632.2004.02324.x.

9. Ceyhan AM, Yildirim M, Basak PY, Akkaya VB, Erturan I. A case of erysipeloid cutaneous leishmaniasis: atypical and unusual clinical variant. Am J Trop Med Hyg. 2008;78(3):406-408. 